\title{
Chitosan films and chitosan/pectin polyelectrolyte complexes encapsulating silver sulfadiazine for wound healing
}

\author{
Gökçen Yașayan' ${ }^{10}$ \\ 'Marmara University, Faculty of Pharmacy, Department of Pharmaceutical Technology, Haydarpașa, İstanbul, Turkey
}

ORCID IDs of the authors: G.Y. 0000-0002-5299-2924

Cite this article as: Yasayan, G. (2020). Chitosan films and chitosan/pectin polyelectrolyte complexes encapsulating silver sulfadiazine for wound healing. Istanbul Journal of Pharmacy. Advanced Online Publication.

\begin{abstract}
Background and Aims: The use of natural polymers as wound dressings is attracting more interest due to their favoured properties such as biodegradability and biocompatibility. With this background, chitosan films and chitosan/pectin polyelectrolyte complex films encapsulating silver sulfadiazine were fabricated as novel wound dressings.

Methods: Films were fabricated, and the surface topography and the surface roughness of the films were characterised by atomic force microscopy. Swelling and hydrolytic degradation behaviours of the films were monitored, and surface chemistry analysis was carried out. Following drug release studies, release kinetics were studied to evaluate the films for drug delivery. Results: Results suggest that the characteristic crystalline structure of chitosan films disappears after complexation with pectin. Polyelectrolyte complex films were found to be more durable than chitosan films due to their improved resistance to hydrolytic degradation. No incompatibilities amongst formulation components were detected. In vitro drug release studies indicated a rapid release of the drug from chitosan films compared to polyelectrolyte complex films.

Conclusion: The overall results suggest that chitosan/pectin polyelectrolyte complex films have improved properties in terms of durability compared to chitosan films. Both films could be a promising candidate for wound healing applications considering the specific needs of different types of wounds.
\end{abstract}

Keywords: Chitosan, silver sulfadiazine, polyelectrolyte

\section{INTRODUCTION}

Wound care is an important topic in the worldwide health care systems for both clinical and economic reasons. Wounds, especially chronic non-healing types, may affect living standards of the patients significantly, and treatment costs of wound therapies cause an economic burden (Gottrup, 2004; Olsson et al., 2019). It has been foreseen that number of people facing wounds will increase as a result of increases in ageing populations, as well as in the incidence rates of patients with chronic diseases such as diabetes and circulatory abnormalities (Lai et al., 2014).

Coping efficiently with wounds involves three main strategies: prevention, diagnosis and treatment. For wound treatment, the type of wound and the function of the wound dressing should be considered in order to facilitate wound healing. Dressings should be selected based on the specific needs of the wound to provide the optimum conditions for healing. These conditions could be adjusting moisture and temperature, enabling gas exchange, promoting new tissue regeneration, protecting against pathogens and other external contaminations. Also being non-toxic, non-allergic, and non-adherent to newly formed granu-

Address for Correspondence:

Gökçen YAȘAYAN, e-mail: gokcen.yasayan@marmara.edu.tr

This work is licensed under a Creative Commons Attribution 4.0 International License. 
lation tissue are favoured (Dhivya, Padma \& Santhini, 2015; Vowden \& Vowden, 2017; Han \& Ceilley, 2017).

Traditional wound dressings such as gauze and cotton wool and modern ones including film, foam, hydrogel, hydrocolloid, alginate, and membrane dressings are used for treatment. Traditional dressings could keep the wound dry and clean, while modern ones have additional functionalities to support wound healing, such as hydrating/dehydrating wound, controlling exudate accumulation and avoiding bacterial contamination. More recent modern dressings include dressings fabricated using biopolymers; most commonly chitosan, pectin, elastin, collagen and hyaluronic acid (Ehterami et al., 2018; Singh \& Han, 2016; Dhivya et al., 2015). These biopolymers are either the main structural elements of the extracellular matrix, or support wound healing due to their characteristic properties (Saghazadeh et al., 2018).

Chitosan is a natural polysaccharide that is commonly used in wound dressings. It uses many mechanisms to promote wound healing; by enhancing granulation, supporting organisation, and accelerating the production of biological mediators, macrophages, and fibroblasts (Ueno, Mori \& Fujinaga, 2001). In addition, this biocompatible and biodegradable polymer has haemostatic, fungistatic, anti-inflammatory, and antimicrobial properties (Jayakumar, Prabaharan, Kumar, Nair, \&Tamura, 2011; Dai, Tanaka, Huang \& Hamblin, 2011). In literature, the use of chitosan alone or in combination with other polymers as polyelectrolyte complexes or blends has been reported (Berger, Reist, Mayer, Felt \& Gurny, 2004).

Pectin is also a polysaccharide that has a low toxicity. They are commonly obtained from the cell walls of plants, such as apple pomace and citrus (Coimbra et al., 2011). It is a natural polymer with anti-inflammatory and anti-carcinogenic properties and it has been demonstrated that modified citrus pectin is effective in many cancer types and stages (Glinsky \& Raz, 2009; Coimbra et al., 2011; Martins, Camargo, Bishop, Popat, Kipper, \& Martins, 2018).

Considering the advantages of chitosan and pectin in wound healing, in this study, we have fabricated a novel wound dressing with the use of chitosan and chitosan/pectin polyelectrolyte complexes encapsulating silver sulfadiazine. Chitosan/ pectin polyelectrolyte complexes (PEC) are formed mainly due to strong electrostatic interactions of cationic chitosan and anionic pectin, between the ionized amino groups of chitosan and the ionized carboxyl acid groups of pectin (Maciel, Yoshida, \& Franco, 2015; Rashidova et al., 2004; Coimbra et al., 2011).

These PEC films have many advantages such as the use of natural polymers, easy fabrication, biocompatibility and biodegradability, and improved mechanical properties (Maciel et al., 2015) Studies with chitosan/pectin PECs are carried for drug delivery applications and as wound dressings in literature (Coimbra et al., 2011; Martins et al., 2018; Bigucci et al., 2008). However, silver sulfadiazine has not encapsulated within these complexes previously.

Silver sulfadiazine is an effective antimicrobial agent, and consists of two agents: silver and sulphonamide. It is remarkably safe, and used mainly in wound dressings, most commonly for the treatment of burns. More recently, silver sulfadiazine has been used to coat medical devices such as catheters to avoid infections in the wound area (Klasen 2000; Kleinbeck, Bader, \& Kao, 2009; White \& Cooper, 2005).

Once the films were fabricated, they were characterised by atomic force microscopy (AFM) and fourier transform infrared (FT-IR) spectroscopy. The swelling behaviour of the films was evaluated, and hydrolytic degradation studies were carried out. Afterwards, the silver sulfadiazine was loaded to formulations and in vitro drug release studies were carried out.

\section{MATERIALS AND METHODS}

\section{Materials}

Silver sulfadiazine was a kind gift from Deva Pharmaceuticals (Turkey). Chitosan (molecular weight 100.000-300.000 Da) was purchased from Acros Organics (US). Pectin (citrus type USP/100) was purchased from Herbstreith and Fox KG. Glacial acetic acid and sodium hydroxide was purchased from Honeywell Riedel-de-Haën (Germany). PPP-NCLR AFM probes were purchased Nanosensors (Switzerland) and all the other reagents used were analytical grade and purchased from SigmaAldrich (Germany).

\section{Fabrication of chitosan films}

Chitosan was dissolved in acetic acid $(10 \mathrm{mg} / \mathrm{mL})$ at room temperature, and afterwards neutralized to pH 6.0 (Llanos, de Oliveira Vercik, \& Vercik, 2015; Tanabe, Okitsu, Tachibana, \& Yamauchi, 2002). The solution was poured over moulds and dried in an oven at $30^{\circ} \mathrm{C}$ (Memmert UM500), and the films were obtained. For preparation of the drug loaded films, silver sulfadiazine was dissolved in the polymer solution at $5 \mathrm{mg} / \mathrm{ml}$, and the procedure described was followed (Yaşayan, Karaca, Akgüner, \& Bal-Öztürk, 2020).

\section{Fabrication of chitosan/pectin polyelectrolyte complex films}

The optimum complexation ratio for chitosan and pectin was selected as 1:4.3 (w:w) at pH 5.5 (Maciel et al., 2015). Briefly, chitosan was dissolved in aqueous acetic acid, while pectin was distilled water using a magnetic stirrer at room temperature. Once the polymers were dissolved, the $\mathrm{pH}$ values of each mixture was adjusted to 5.5. The chitosan suspension was then added to pectin suspension slowly by a micropipette under stirring, and mixed for 30 minutes using a magnetic stirrer to obtain PECs. The PECs were then centrifuged at 12000 rpm for 30 minutes at $4{ }^{\circ} \mathrm{C}$ (Beckman Coulter Allegra X-30R Centrifuge) to be separated from the unreacted chitosan and pectin remaining. Finally, the PEC precipitate was placed in petri dishes, and dried in a drying oven at $37^{\circ} \mathrm{C}$ (Maciel et al., 2015), and the films were obtained. For preparation of the drug loaded films, silver sulfadiazine was added to chitosan solution $(5 \mathrm{mg} / \mathrm{ml}$ ) under magnetic stirring at room temperature, and the procedure described was followed (Yaşayan et al., 2020).

\section{Topographical investigations}

Topography images of the films were obtained using an Ambient AFM ${ }^{\text {TM }}$ (Nanomagnetics Instruments). The AFM study was 
carried in air, operating in dynamic mode at scan rates between 0.5-1.5 Hz. PPP-NCLR AFM probes (nominal resonance frequency: $190 \mathrm{kHz}$, nominal force constant: $48 \mathrm{~N} / \mathrm{m}$ ) were used. Image data was analysed by a NMI Image Analyzer v1.5 (Nanomagnetics Instruments). Using the image data, surface roughness analyses were carried out.

\section{Swelling studies}

Films were cut into square pieces. Accurately weighted dry films were immersed in phosphate buffer saline (PBS) at pH 7.4 at 37 ${ }^{\circ} \mathrm{C}$. At predetermined time points, scaffolds were taken out of the buffer, and weighed immediately after the gentle removal of the water droplets on their surface. Swelling percentages were calculated using the following formula (Yaşayan et al., 2020):

$$
\text { Swelling }(\%)=\frac{W s-W d}{W d} \times 100
$$

where Ws is the weight of swollen scaffold, and Wd is the weight of dried scaffold.

\section{Hydrolytic degradation studies}

Accurately weighted dry films were immersed in PBS at pH 7.4 and placed in a shaking water bath at $37^{\circ} \mathrm{C}$. At predetermined time points, scaffolds were taken out of buffer, dried under a fume hood, and weighed. The weight remaining percentages of the films were calculated using the following formula given (Yaşayan et al., 2020):

$$
\text { Weight remaining }(\%)=\frac{W_{\text {after }}}{W_{\text {before }}} \times 100
$$

where $W_{\text {before }}$ is the weight of a dried scaffolds before hydrolytic degradation, and $\mathrm{W}_{\text {after }}$ is the weight after hydrolytic degradation.

\section{Surface chemistry analysis}

Fourier transform infrared spectroscopy (FT-IR) studies were carried out using a Shimadzu IR Prestige 21 spectrometer in the range of $4000-700 \mathrm{~cm}^{-1}$. The studies were carried out to detect any chemical incompatibilities between the formulation components.

\section{Encapsulation efficiency and in vitro drug release studies}

Drug-loaded films were cut into pieces, and placed into glacial acetic acid and PBS containing capped glass vials using a shaking bath. Once the samples dissolved, the supernatant was centrifuged. Following a pH adjustment, the supernatant was diluted suitably, and quantified using a UV spectrophotometer (BioTek Epoch microplate reader, BioTek Instruments, Inc.) at $300 \mathrm{~nm}$. Encapsulation efficiency was calculated using the equation given below:

$$
\text { Encapsulation efficiency }(\%)=\frac{\text { Actual drug content }}{\text { Theoretical drug content }} \times 100
$$

For in vitro drug release studies, films were immersed in screw capped vials filled with $20 \mathrm{ml}$ of PBS medium at pH 7.4. Dissolution studies were carried out using a water bath shaker at 37 ${ }^{\circ} \mathrm{C}$ for 24 hours under sink conditions. At predetermined time points, samples were withdrawn for analysis, and the same vol- ume of fresh PBS medium was added to vials to maintain the total dissolution volume after each withdrawal. The amount of released drug was measured spectroscopically at $300 \mathrm{~nm}$ using a UV spectrophotometer (BioTek Epoch microplate reader, BioTek Instruments, Inc.). The results were calculated cumulatively (Kleinbeck et al., 2009).

The drug release mechanisms were determined by applying kinetic models. The cumulative drug release data were fitted to zero-order, first-order, Higuchi, Korsemeyer-Peppas, and Hixson-Crowell models. The determination coefficient $\left(R^{2}\right)$ value was calculated by fitting $60 \%$ of the linear regression data of release profiles, and the best fit model was determined by evaluation of $\mathrm{R}^{2}$ value proximity to 1 (England, Miller, Kuttan, Trent, \& Frieboes, 2015)

\section{Statistical analysis}

Statistical analyses of the data were performed by a t-test. P < 0.05 was considered statistically significant.

\section{RESULTS AND DISCUSSION}

In this study, chitosan films and chitosan/pectin PEC films were fabricated by casting and solvent evaporation methods. In literature, the optimum complexation ratio for chitosan and pectin is given as 1:4.3 (w:w) at pH 5.5 to obtain the highest product yield (Maciel et al., 2015). The pKa value of pectin is in the range of 3.5-4.5, and the pKa value of chitosan is around 6.2-7.0 (Maciel et al., 2015). Considering the pKa values of polymers, PECs of chitosan and pectin are prepared at $\mathrm{pH} 5.5$, in between the pKa values of these two polymers where both polymers have a high ratio of ionised groups for ionic interaction (Coimbra et al., 2011; Rashidova et al., 2004; Bigucci et al., 2008). Therefore, the complexation of PEC films was conducted using these parameters. After fabrication, silver sulfadiazine was loaded to films to further support wound healing.

AFM topography images of films before and after drug loading is given in Figure 1. For the chitosan films (Figure 1a), characteristic crystalline structure of the chitosan could be observed from image data (Lewandowska, Sionkowska, \& Grabska, 2015;
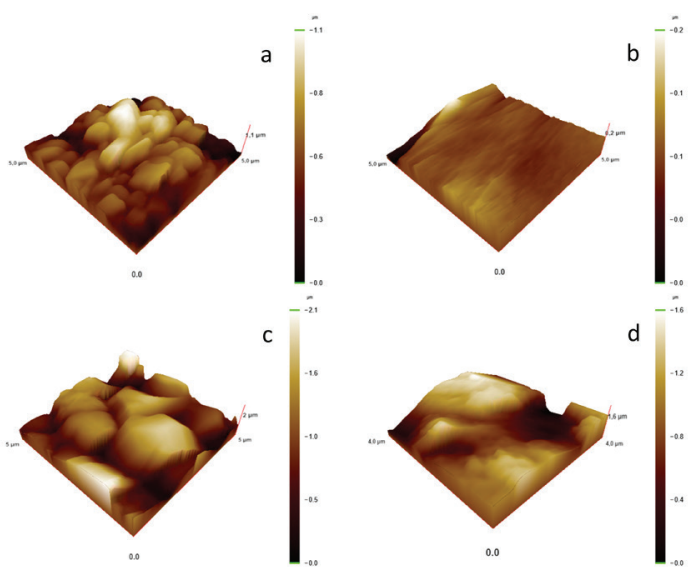

Figure 1. AFM topography images of films before and after drug loading. Chitosan films (a), chitosan-pectin PEC films (b), silver sulfadiazine loaded chitosan films (c), silver sulfadiazine loaded chitosan-pectin PEC films (d). 
Rinaudo, 2006). After formation of the PECs with pectin (Figure $1 \mathrm{~b})$, the chitosan's characteristic structure disappeared, and relatively flat surfaces were observed. In literature, flat, smooth and homogeneous surface morphology is reported for chitosan/pectin PEC films as the indicator of complex formation with a strong interaction (Maciel et al., 2015). Surface roughness values were calculated from AFM image data, and the results were given in Table 1 . Indeed, the surface roughness of the crystalline chitosan films was found to be relatively high compared to smooth PEC films. The average roughness value for the chitosan films was found to be $177.58 \mathrm{~nm}$, whereas for the chitosan-pectin PEC films it was $10.73 \mathrm{~nm}$.

The surface roughness values calculated from the image data for drug loaded surfaces was found to be $344.20 \mathrm{~nm}$ for chitosan films and $300.56 \mathrm{~nm}$ for PEC films (Table 1). It was observed that the roughness values increased after the addition of the drug.

\section{Table 1. Average roughness values of chitosan films and chitosan-pectin polyelectrolyte complex (PEC) films before and after drug loading. The roughness values were calculated using AFM image data from Figure 1.}

\begin{tabular}{|c|c|}
\hline Sample & $\begin{array}{l}\text { Average Roughness (Ra) } \\
\text { (nm) }\end{array}$ \\
\hline \multicolumn{2}{|c|}{ Films } \\
\hline Chitosan films & 177.58 \\
\hline Chitosan-pectin PEC films & 10.73 \\
\hline \multicolumn{2}{|c|}{ Silver sulfadiazine loaded films } \\
\hline Chitosan films & 344.20 \\
\hline Chitosan-pectin PEC films & 300.56 \\
\hline
\end{tabular}

After drug loading, the morphology of surfaces changed dramatically, and characteristic morphologies of the chitosan films and the PEC films disappeared (Figure 1c-d), Silver sulfadiazine loaded chitosan films were observed to have large microdomains probably due to molecular interactions between the chitosan and the drug. For PEC films, the smooth structure disappeared after drug loading, and the drug loaded surface was characterized with holes and microdomains.

After morphological investigations, the films were characterised by swelling and hydrolytic degradation studies. Wound dressings should provide a moist environment to enhance wound healing. Thus swelling, and gel formation over the wound is desired for wound dressings. This gel provides occlusive conditions due to gel formation, where the gel acts as a barrier. Also an ideal wound dressing should absorb the exudate of the wound (Boateng, Matthews, Stevens, \& Eccleston, 2008).

Swelling studies were performed by accurately weighting the dry films, and immersing them into PBS buffer at $\mathrm{pH} 7.4$ at 37 ${ }^{\circ} \mathrm{C}$, and the results are given in Figure $2 \mathrm{a}$. According to the results, after 30 minutes, both films were attained an equilibrium state. After the $30^{\text {th }}$ minute, it was observed that the swelling ratio of the chitosan films was higher compared to the PEC films. The swelling ratio was in the range of $650-700 \%$ for the chitosan films, and in the range of $230-260 \%$ for the PEC films. a

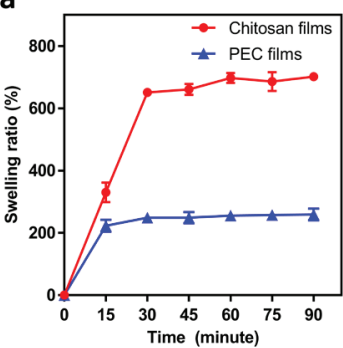

b

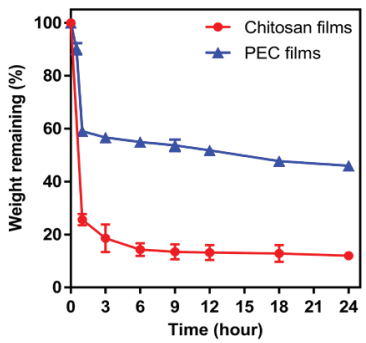

Figure 2. Swelling studies (a) and hydrolytic degradation studies (b) of chitosan films and chitosan-pectin PEC films.

The hydrolytic degradation results are given in Figure $2 \mathrm{~b}$. According to the results, it was observed that the hydrolytic degradation rates of films were reduced by complexation of chitosan with pectin. For the chitosan films, the remaining weight percentage was $26 \%$ after the $1^{\text {st }}$ hour, and reduced to $12 \%$ by the $24^{\text {th }}$ hour. For the PEC films, the reduction of weight loss was not as fast as the chitosan films; the remaining weight percentage was $60 \%$ after the $1^{\text {st }}$ hour, and reduced to $46 \%$ by the $24^{\text {th }}$ hour. The results indicate that polyelectrolyte complex formation between the polymers has improved the mechanical stability and durability of the films significantly; and by PEC formation, rapid degradation of chitosan is eliminated.

Comparative FT-IR spectra of the components in the chitosan film formulation are given in Figure 3. Chitosan has typical bands for polysaccharides in the range of $3400-2800 \mathrm{~cm}^{-1}$. Characteristic peaks of chitosan are observed at $3351 \mathrm{~cm}^{-1}, 2940 \mathrm{~cm}^{-1}, 2879$ $\mathrm{cm}^{-1}$ that corresponds to the stretching vibrations of $-\mathrm{NH}$ and $-\mathrm{OH}$ groups, aliphatic $-\mathrm{CH}_{2}$ groups, and aliphatic $-\mathrm{CH}_{3}$ groups respectively. At $1656 \mathrm{~cm}^{-1}$ peak of amide I ( $\mathrm{C}=\mathrm{O}$ band), and at $1586 \mathrm{~cm}^{-1}$ amide II band, $\mathrm{N}-\mathrm{H}$ bending vibrations are observed

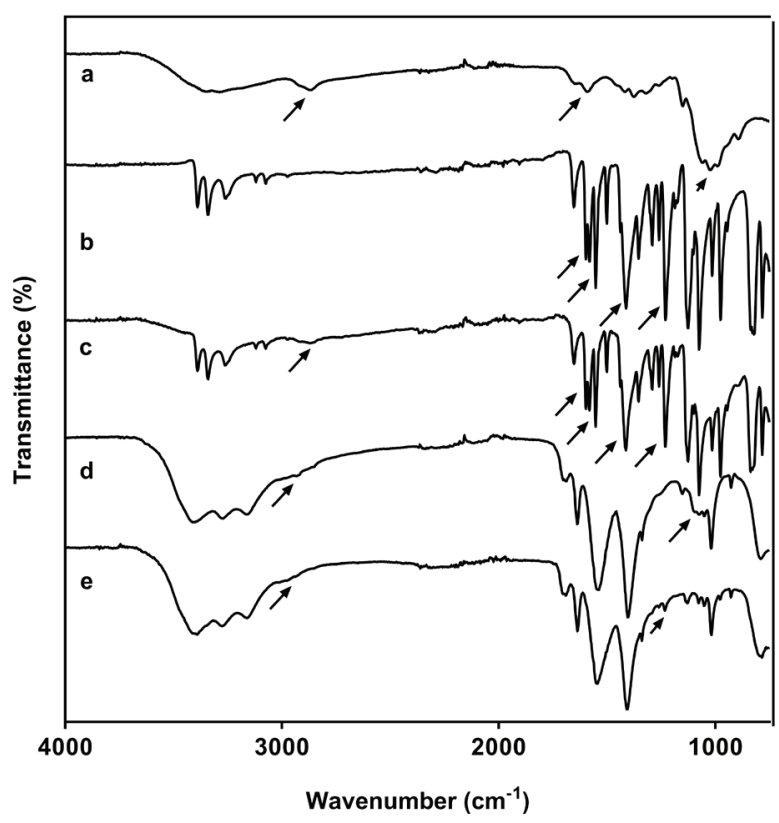

Figure 3. Comparative FT-IR spectra of the components in the chitosan film formulation. Chitosan (a), silver sulfadiazine (b), physical mixture of chitosan and silver sulfadiazine (c), chitosan films before drug loading (d), chitosan films after drug loading (e). 
(Yaşayan et al., 2020) (Figure 3a). For silver sulfadiazine, silver ion is in coordination with various functional groups within the molecule; these groups are deprotonated amine nitrogen, sulfonyl oxygen, the pyrimidine nitrogen in the same chain. It is also in coordination with the further pyrimidine nitrogen. The characteristics bands are at 1596 and at $1583 \mathrm{~cm}^{-1}$ for phenyl skeletal vibrations conjugated to the $\mathrm{NH}_{2}$ group and the aromatic ring's C-C stretching vibrations. The bands at 1556, 1503 and 1412 $\mathrm{cm}^{-1}$ correspond to pyrimidine skeletal vibrations (Szegedi et al., 2014). The bands at 3340 and $3389 \mathrm{~cm}^{-1}$ correspond to $-\mathrm{NH}_{2}$ stretching vibrations (Shao et al., 2017). The peak at $1233 \mathrm{~cm}^{-1}$ was attributed to the asymmetrical $\mathrm{S}=\mathrm{O}$ stretching vibration (Shao et al., 2017) (Figure 3b). For the data of physical mixture of chitosan and silver sulfadiazine, characteristic bands of silver sulfadiazine are observed clearly from the data (Figure 3c). For the chitosan films, it was observed that characteristic peaks of pure chitosan were shifted due to use of acetic acid to prepare films, and then the neutralization of chitosan solution by $\mathrm{NaOH}$. In literature it is reported that deprotonation of amine groups results in peak shifts (Takara, Marchese, \& Ochoa, 2015). The band at 1645 is attributed to the asymmetric and symmetric bending vibrations of the $\mathrm{NH}^{3+}$ (Coimbra et al., 2011) (Figure 3d). For the drug loaded chitosan films, the characteristic peaks of the drug were detected in lower intensities, however the presence of the drug is evident at $1233 \mathrm{~cm}^{-1}$. It was observed that the characteristic band of the silver sulfadiazine at $1597 \mathrm{~cm}^{-1}$ was shifted to $1645 \mathrm{~cm}^{-1}$. This is probably as a result of the formation of hydrogen bonds among the amino groups of chitosan and silver sulfadiazine, which resulted in shift of the band to a higher wavenumber, and a reduction in the band intensities (Fajardo et al, 2013). When characteristic bands of silver sulfadiazine are considered, the data suggests that no undesired interactions are observed between the drug and the polymers (Figure 3e).

FTIR spectra of chitosan and silver sulfadiazine were again inserted to Figure 4 in order to better evaluate any possible interactions amongst the formulation components (Figure 4a, c). Pectin has characteristic bands in the range of $3400-2800 \mathrm{~cm}^{-1}$ that correspond to polysaccharides. The peak in the range of at $1727 \mathrm{~cm}^{-1}$ corresponds to the $\mathrm{C}=\mathrm{O}$ stretching vibrations of the methyl ester group and the un-dissociated carboxyl group, and at $1606 \mathrm{~cm}^{-1}$ to the $\mathrm{C}=\mathrm{O}$ asymmetric stretching vibrations and the carboxylate group (Bigucci et al., 2008; Maciel et al., 2015) (Figure 4b). For the data of physical mixture of the polymers and silver sulfadiazine, characteristic bands of silver sulfadiazine are observed clearly at $1233 \mathrm{~cm}^{-1}$ and at $1596 \mathrm{~cm}^{-1}$ (Figure $4 \mathrm{~d}$ ). For PEC films, shifts in amine group of chitosan to $1559 \mathrm{~cm}^{-1}$ could be related with the formation of complexes. When the characteristic bands of chitosan and pectin are considered, the characteristic bands of PEC films are changed due to an ionic interaction of protonated amino groups of chitosan and the carboxyl groups of pectin. Also other groups in the polymers may interact, such as $-\mathrm{OH}$ groups (Figure 4e). For drug loaded PEC films, the characteristic peaks of the drug were detected in lower intensities; probably due to lower amounts of the drug compared to polymer amounts. Similar to chitosan films, the presence of the drug is evident at 1233 $\mathrm{cm}^{-1}$ It was observed that the characteristic band of the silver sulfadiazine at $1597 \mathrm{~cm}^{-1}$ was shifted to $1645 \mathrm{~cm}^{-1}$, probably

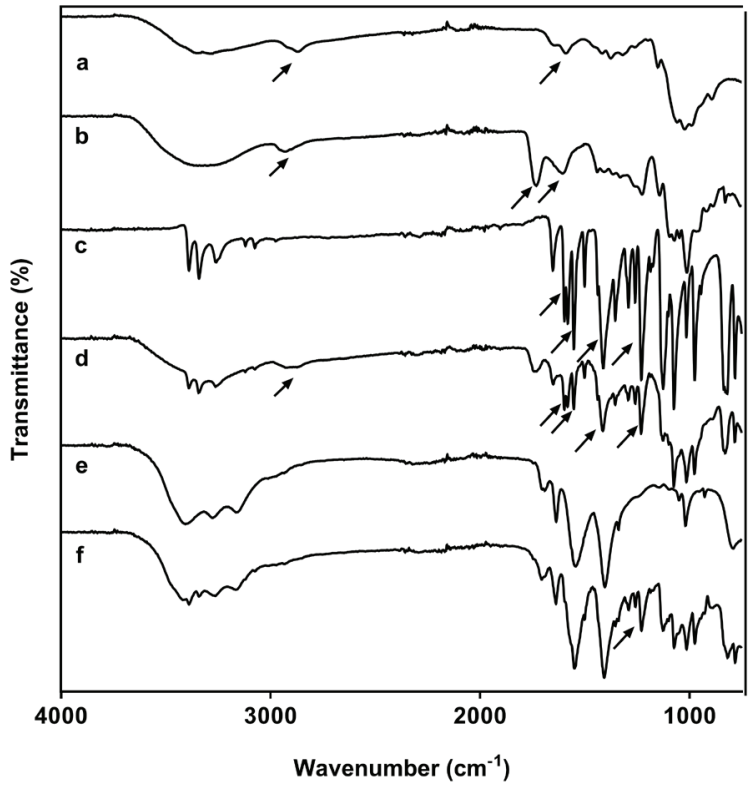

Figure 4. Comparative FT-IR spectra of the components in the PEC film formulation. Chitosan (a), pectin (b), silver sulfadiazine (c), physical mixture of chitosan, pectin, and silver sulfadiazine (d), PEC films before drug loading (e), PEC films after drug loading (f).

as a result of a hydrogen bond formation among the amino groups of chitosan and silver sulfadiazine (Fajardo et al, 2013). (Figure 4f). From the overall FT-IR data, undesired interactions were not detected between the formulation components.

When FT-IR data is evaluated together with AFM data, change in surface topography and the formation of microdomains in drug loaded films could be a result of hydrogen bonds among silver sulfadiazine and chitosan.

Encapsulation efficiencies of the films were studied briefly by cutting the films into small pieces, dissolving them using acetic acid, and quantifying the drug amount in the supernatant. According to the results, encapsulation efficiencies of the drug were $>$ >95\% for the chitosan films and >90\% for the PEC films.

In vitro drug release studies were performed in $\mathrm{PBS}$ at $37^{\circ} \mathrm{C}, \mathrm{pH}$ 7.4, and the results are given in Figure 5. According to the re-

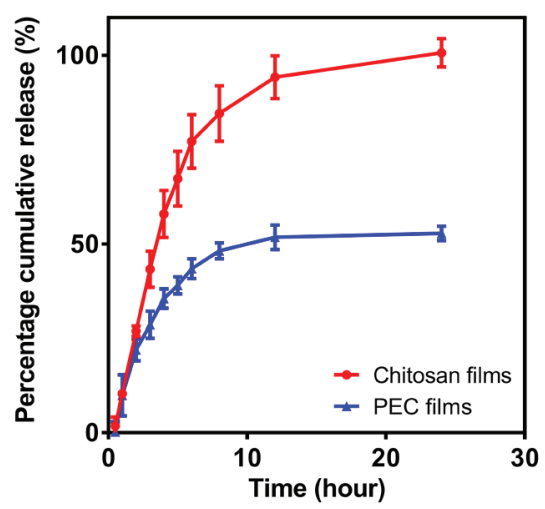

Figure 5. In vitro drug release studies of chitosan films and chitosanpectin PEC films. 
Table 2. Drug release kinetics of the chitosan (CS) films and polyelectrolyte complex (PEC) films.

\begin{tabular}{|lccccccccccccc} 
& \multicolumn{2}{c}{ Zero order } & \multicolumn{2}{c}{ First order } & \multicolumn{2}{c}{ Higuchi } & \multicolumn{2}{c}{ Korsmeyer Peppas } & \multicolumn{2}{c}{ Hixson-Crowell } \\
\hline Films & $\boldsymbol{R}^{2}$ & $\begin{array}{c}K_{0} \\
\left(\boldsymbol{h}^{-1}\right)\end{array}$ & $\boldsymbol{R}^{2}$ & $\begin{array}{c}\boldsymbol{K}_{\mathbf{1}} \\
\left(\boldsymbol{h}^{-1}\right)\end{array}$ & $\boldsymbol{R}^{2}$ & $\boldsymbol{K}_{\mathrm{H}}\left(\boldsymbol{h}^{-1 / 2}\right)$ & $\boldsymbol{R}^{2}$ & $\boldsymbol{n}$ & $\begin{array}{c}\boldsymbol{K}_{\mathrm{KP}} \\
\left(\boldsymbol{h}^{-n}\right)\end{array}$ & $\boldsymbol{R}^{2}$ & $K_{\mathrm{HC}}\left(\boldsymbol{h}^{-1 / 3}\right)$ \\
\hline CS & $\mathbf{0 . 9 9 9}$ & 16.12 & 0.992 & -0.105 & 0.988 & 43.642 & 0.966 & 1.639 & 7.333 & 0.997 & 0.324 \\
PEC & 0.566 & 1.529 & 0.631 & -0.011 & 0.763 & 10.726 & $\mathbf{0 . 8 3 3}$ & 0.517 & 14.598 & 0.61 & 0.033 \\
\hline
\end{tabular}

Zero order, first-order, Higuchi, Korsmeyer Peppas, and Hixson-Crowell models were applied to evaluate drug release kinetics. The R² values, release rate constants $(K)$, and for Korsmeyer Peppas model, release exponent $(n)$ values were calculated.

sults, it was observed that polyelectrolyte complex formation between the polymers affect the cumulative release of the drugs from the films. From the data, the chitosan films released all of the drug at the end of 24 hours, while the PEC films released $52 \%$ of the drug loaded within 24 hours. This drug release at a slower rate with the PEC films could have advantages especially for slow-healing wounds.

After the in vitro drug release studies, mathematical models were applied to drug release profiles to study drug release mechanisms. The $R^{2}$ values were calculated by fitting the first $60 \%$ of the linear regression data of the cumulative drug release profiles (England et al., 2015). During the studies, zero order, first-order, Higuchi, Korsmeyer Peppas, and Hixson-Crowell models were applied to evaluate drug release kinetics. The best-fit model was selected by a proximity of $R^{2}$ value to 1 . The $R^{2}$ values and release rate constants $(K)$, and the release exponent $(n)$ values for the Korsmeyer Peppas model are given in Table 2. The results indicated that chitosan films best fit into the zero order model, whereas PEC films fit into the Korsmeyer Peppas model. Zero order kinetics indicates a constant drug release from the chitosan films. For Korsmeyer Peppas model, the $n$ value is calculated to evaluate the release mechanism. It is well known that the drug transport mechanism is Fickian diffusion when the $n$ value is 0.5 , non-Fickian transport when $0.45<n=0.89$, Case II transport when the $n$ value is 0.89 , super case II transport when the $n$ value is higher than 0.89 (Gouda et al., 2017). In our results, the $n$ value was found to be 0.517 , which indicates the drug release mechanism is non-Fickian transport (Gouda, Raishya, \& Qing, 2017).

\section{CONCLUSION}

In this study, chitosan films and chitosan/pectin PEC wound dressings were fabricated encapsulating silver sulfadiazine by casting and solvent evaporation methods. The ease of fabrication, having favoured properties of polymers in wound healing, and additional benefits of these polymers that promote wound healing are the aims of our choice. These films are designed to improve wound healing, and for this purpose silver sulfadiazine is loaded in the films to further support wound healing by antimicrobial properties of the drug. We found that by formation of polyelectrolyte complexes, mechanical properties and durability of the films could be improved, and drug release could be extended compared to chitosan films. Future studies should be conducted to fully evaluate the films in terms of wound healing properties and antimicrobial properties including in-vivo studies. We hope these wound dressings may have a potential application as wound dressings, and this study could be useful for the fabrication of novel dressings that replace conventional dressings.

Acknowledgement: We would like to thank to Deva Pharmaceuticals (Turkey) for providing silver sulfadiazine as gift.

Peer-review: Externally peer-reviewed.

Author Contributions: Conception/Design of Study- G.Y.; Data Acquisition- G.Y;; Data Analysis/Interpretation- G.Y.; Drafting Manuscript- G.Y.; Critical Revision of Manuscript- G.Y.; Final Approval and AccountabilityG.Y.; Technical or Material Support- G.Y.; Supervision- G.Y.

Conflict of Interest: The authors have no conflict of interest to declare.

Financial Disclosure: Authors declared no financial support.

\section{REFERENCES}

- $\quad$ Berger, J., Reist, M., Mayer, J. M., Felt, O., \& Gurny, R. (2004). Structure and interactions in chitosan hydrogels formed by complexation or aggregation for biomedical applications. European Journal of Pharmaceutics and Biopharmaceutics, 57(1), 35-52.

- $\quad$ Bigucci, F., Luppi, B., Cerchiara, T., Sorrenti, M., Bettinetti, G., Rodriguez, L., \& Zecchi, V. (2008). Chitosan/pectin polyelectrolyte complexes: selection of suitable preparative conditions for colonspecific delivery of vancomycin. European Journal of Pharmaceutical Sciences, 35(5), 435-441.

- Boateng, J. S., Matthews, K. H., Stevens, H. N. E., \& Eccleston, G. M. (2008). Wound healing dressings and drug delivery systems: A review. Journal of Pharmaceutical Sciences, 97(8), 2892-2923. http:// dx.doi.org/10.1002/jps.21210

- Coimbra, P., Ferreira, P., De Sousa, H., Batista, P., Rodrigues, M., Correia, I., \& Gil, M. (2011). Preparation and chemical and biological characterization of a pectin/chitosan polyelectrolyte complex scaffold for possible bone tissue engineering applications. International Journal of Biological Macromolecules, 48(1), 112-118.

- Dai, T., Tanaka, M., Huang, Y. Y., \& Hamblin, M. R. (2011). Chitosan preparations for wounds and burns: antimicrobial and woundhealing effects. Expert Review of Anti-infective Therapy, 9(7), 857879. http://dx.doi.org/10.1586/eri.11.59

Dhivya, S., Padma, V. V., \& Santhini, E. (2015). Wound dressings a review. BioMedicine, 5(4), 22-22. http://dx.doi.org/10.7603/ s40681-015-0022-9

- $\quad$ Ehterami, A., Salehi, M., Farzamfar, S., Vaez, A., Samadian, H., Sahrapeyma, H. ... Goodarzi, A. (2018). In vitro and in vivo study of PCL/COLL wound dressing loaded with insulin-chitosan nanoparticles on cutaneous wound healing in rats model. International Journal of Biological Macromolecules, 117, 601-609. http://dx.doi.org/10.1016/j.ijbiomac.2018.05.184 
- $\quad$ England, C. G., Miller, M. C., Kuttan, A., Trent, J. O., \& Frieboes, H. B. (2015). Release kinetics of paclitaxel and cisplatin from two and three layered gold nanoparticles. European Journal of Pharmaceutics and Biopharmaceutics, 92, 120-129. http://dx.doi. org/10.1016/j.ejpb.2015.02.017

- Fajardo, A. R., Lopes, L. C., Caleare, A. O., Britta, E. A., Nakamura, C. V., Rubira, A. F., \& Muniz, E. C. (2013). Silver sulfadiazine loaded chitosan/chondroitin sulfate films for a potential wound dressing application. Materials Science and Engineering: C, 33(2), 588-595. https://doi.org/10.1016/j.msec.2012.09.025

- Glinsky, V. V., \& Raz, A. (2009). Modified citrus pectin anti-metastatic properties: one bullet, multiple targets. Carbohydrate Research, 344(14), 1788-1791. http://dx.doi.org/10.1016/j. carres.2008.08.038

- Gottrup, F. (2004). A specialized wound-healing center concept: importance of a multidisciplinary department structure and surgical treatment facilities in the treatment of chronic wounds. The American Journal of Surgery, 187(5, Supplement 1), S38-S43. http://dx.doi.org/10.1016/S0002-9610(03)00303-9

- Gouda, R., Baishya, H., \& Qing, Z. (2017). Application of mathematical models in drug release kinetics of carbidopa and levodopa ER tablets. Journal of Developing Drugs, 6(02).

- Han, G., \& Ceilley, R. (2017). Chronic Wound Healing: A Review of Current Management and Treatments. Advances in therapy, 34(3), 599-610. http://dx.doi.org/10.1007/s12325-017-0478-y

- Llanos, J. H. R., de Oliveira Vercik, L. C., \& Vercik, A. (2015). Physical properties of chitosan films obtained after neutralization of polycation by slow drip method. Journal of Biomaterials and Nanobiotechnology, 6(04), 276.

- Jayakumar, R., Prabaharan, M., Kumar, P. T. S., Nair, S. V., \& Tamura, H. (2011). Biomaterials based on chitin and chitosan in wound dressing applications. Biotechnology Advances, 29(3), 322-337. http://dx.doi.org/10.1016/j.biotechadv.2011.01.005

- Klasen, H. J. (2000). A historical review of the use of silver in the treatment of burns. II. Renewed interest for silver. Burns, 26(2), 131-138. http://dx.doi.org/10.1016/S0305-4179(99)00116-3

- Kleinbeck, K. R., Bader, R. A., \& Kao, W. J. (2009). Concurrent in vitro release of silver sulfadiazine and bupivacaine from semi-interpenetrating networks for wound management. Journal of burn care \& research: Official publication of the American Burn Association, 30(1), 98-104. http://dx.doi.org/10.1097/BCR.0b013e3181921ed9

- Lai, H. J., Kuan, C. H., Wu, H. C., Tsai, J. C., Chen, T. M., Hsieh, D. J., \& Wang, T.W. (2014). Tailored design of electrospun composite nanofibers with staged release of multiple angiogenic growth factors for chronic wound healing. Acta Biomaterialia, 10(10), 4156-4166.

- Lewandowska, K., Sionkowska, A., \& Grabska, S. (2015). Chitosan blends containing hyaluronic acid and collagen. Compatibility behaviour. Journal of Molecular Liquids, 212, 879-884. http:// dx.doi.org/10.1016/j.molliq.2015.10.047

- Maciel, V. B. V., Yoshida, C. M., \& Franco, T.T. (2015). Chitosan/pectin polyelectrolyte complex as a pH indicator. Carbohydrate Polymers, $132,537-545$
Martins, J. G., Camargo, S. E., Bishop, T. T., Popat, K. C., Kipper, M. J., \& Martins, A. F. (2018). Pectin-chitosan membrane scaffold imparts controlled stem cell adhesion and proliferation. Carbohydrate Polymers, 197, 47-56.

- Olsson, M., Järbrink, K., Divakar, U., Bajpai, R., Upton, Z., Schmidtchen, A., \& Car, J. (2019). The humanistic and economic burden of chronic wounds: A systematic review. Wound Repair and Regeneration, 27(1), 114-125. http://dx.doi.org/10.1111/wrr.12683

Rashidova, S. S., Milusheva, R. Y., Semenova, L., Mukhamedjanova, M. Y., Voropaeva, N., Vasilyeva, S., ... Ruban, I. (2004). Characteristics of interactions in the pectin-chitosan system. Chromatographia, 59(11-12), 779-782.

Rinaudo, M. (2006). Chitin and chitosan: Properties and applications. Progress in Polymer Science (Oxford), 31(7), 603-632. http:// dx.doi.org/10.1016/j.progpolymsci.2006.06.001

Saghazadeh, S., Rinoldi, C., Schot, M., Kashaf, S. S., Sharifi, F., Jalilian, E., ... Khademhosseini, A. (2018). Drug delivery systems and materials for wound healing applications. Advanced Drug Delivery Reviews, 127, 138-166. http://dx.doi.org/10.1016/j.addr.2018.04.008 Shao, W., Wu, J., Wang, S., Huang, M., Liu, X., \& Zhang, R. (2017). Construction of silver sulfadiazine loaded chitosan composite sponges as potential wound dressings. Carbohydrate Polymers, 157, 1963-1970. http://dx.doi.org//10.1016/j.carbpol.2016.11.087 Singh, D., \& Han, S. S. (2016). 3D Printing of Scaffold for Cells Delivery: Advances in Skin Tissue Engineering. Polymers, 8(1). http:// dx.doi.org/10.3390/polym8010019

- $\quad$ Szegedi, Á., Popova, M., Yoncheva, K., Makk, J., Mihály, J., \& Shestakova, P. (2014). Silver- and sulfadiazine-loaded nanostructured silica materials as potential replacement of silver sulfadiazine. Journal of Materials Chemistry B, 2(37), 6283-6292. http://dx.doi. org/10.1039/C4TB00619D

- Takara, E. A., Marchese, J., \& Ochoa, N. A. (2015). NaOH treatment of chitosan films: Impact on macromolecular structure and film properties. Carbohydrate Polymers, 132, 25-30. http://dx.doi. org/10.1016/j.carbpol.2015.05.077

- Tanabe, T., Okitsu, N., Tachibana, A., \& Yamauchi, K. (2002). Preparation and characterization of keratin - chitosan composite film. Biomaterials, 23(May 2001), 817-825.

- Ueno, H., Mori, T., \& Fujinaga, T. (2001). Topical formulations and wound healing applications of chitosan. Advanced Drug Delivery Reviews, 52(2), 105-115. http://dx.doi.org/10.1016/S0169409X(01)00189-2

- Vowden, K., \& Vowden, P. (2017). Wound dressings: principles and practice. Surgery (Oxford), 35(9), 489-494.

White, R., \& Cooper, R. (2005). Silver sulphadiazine: a review of the evidence. Wounds UK, 1(2), 51.

- Yaşayan, G., Karaca, G., Akgüner, Z. P., \& Bal-Öztürk, A. (2020). Chitosan/collagen composite films as wound dressings encapsulating allantoin and lidocaine hydrochloride. International Journal of Polymeric Materials and Polymeric Biomaterials. Advance online publication. https://doi.org/10.1080/00914037.2020.1740993 